Vietnam Journal of Mechanics, VAST, Vol.41, No. 4 (2019), pp. 319-336

DOI: https://doi.org/10.15625/0866-7136/14098

\title{
DYNAMIC RESPONSES OF AN INCLINED FGSW BEAM TRAVELED BY A MOVING MASS BASED ON A MOVING MASS ELEMENT THEORY
}

\author{
Tran Thi Thom ${ }^{1,2, *}$, Nguyen Dinh Kien ${ }^{1,2}$, Le Thi Ngoc Anh ${ }^{3}$ \\ ${ }^{1}$ Institute of Mechanics, VAST, 18 Hoang Quoc Viet, Hanoi, Vietnam \\ ${ }^{2}$ Graduate University of Science and Technology, VAST, 18 Hoang Quoc Viet, Hanoi, Vietnam \\ ${ }^{3}$ Institute of Applied Information and Mechanics, Ho Chi Minh City, Vietnam \\ *E-mail: thomtt0101@gmail.com
}

Received: 02 August 2019 / Published online: 14 November 2019

\begin{abstract}
Dynamic analysis of an inclined functionally graded sandwich (FGSW) beam traveled by a moving mass is studied. The beam is composed of a fully ceramic core and two skin layers of functionally graded material (FGM). The material properties of the FGM layers are assumed to vary in the thickness direction by a power-law function, and they are estimated by Mori-Tanaka scheme. Based on the first-order shear deformation theory, a moving mass element, taking into account the effect of inertial, Coriolis and centrifugal forces, is derived and used in combination with Newmark method to compute dynamic responses of the beam. The element using hierarchical functions to interpolate the displacements and rotation is efficient, and it is capable to give accurate dynamic responses by small number of the elements. The effects of the moving mass parameters, material distribution, layer thickness ratio and inclined angle on the dynamic behavior of the FGSW beam are examined and highlighted.
\end{abstract}

Keywords: inclined FGSW beam; hierarchical functions; moving mass element; MoriTanaka scheme; dynamic responses.

\section{INTRODUCTION}

Sandwich beams are widely used in the aerospace industry as well as in other industries due to their high stiffness to weight ratio. Functionally graded materials (FGMs), initiated by Japanese scientists in 1984, are employed to fabricate functionally graded sandwich (FGSW) beams to improve their performance in severe conditions. Investigations on mechanical behavior of the FGSW beams have been recently reported by several researchers. Bhangale and Ganesan [1] studied thermo-elastic buckling and vibration behavior of a FGSW beam having constrained viscoelastic core using a finite element formulation. Amirani et al. [2] analyzed free vibration of sandwich beam with FGM core 
by a mesh-less method. Bui et al. [3] proposed a novel truly mesh-free radial point interpolation method to investigate transient responses and natural frequencies of sandwich beams with FGM core. Using a mesh-free boundary-domain integral equation method, Yang et al. [4] studied free vibration of the FGSW beams. Based on a refined shear deformation theory and a quasi-3D theory, Vo et al. $[5,6]$ derived finite element formulations for free vibration and buckling analyses of FGSW beams. Nguyen et al. [7] obtained an analytical solution for buckling and vibration analysis of FGSW beams using a quasi-3D shear deformation theory. Again, a quasi-3D theory is used by Vo et al. [8] to study static behavior of FGSW beams. Finite element model and Navier solutions are developed by the authors to determine the displacements and stresses of FGSW beams with various boundary conditions. Su et al. [9] considered free vibration of FGSW beams resting on a Pasternak elastic foundation. The effective material properties of FGM are estimated by both Voigt model and Mori-Tanaka scheme, and the governing equations are solved using the modified Fourier series method. Based on Timoshenko beam theory, Şimşek and Al-shujairi [10] examined static, free and forced vibration of FGSW beams under the action of two moving harmonic loads. The equations of the motion are obtained by the authors using Lagrange's equations, and they are solved by the implicit Newmark- $\beta$ method.

The problem of beams traveled by a moving mass has drawn much attention from scientists [11-15]. The inertial effects of the moving mass including Coriolis, inertia and centrifugal forces are taken into consideration by the authors. Most of the works, however considered the horizontal beams. When the beams are inclined, then the approaches presented in the foregoing researches cannot be directly applied to solve the problem. For this reason, $\mathrm{Wu}[16]$ used the theory of moving mass element to determine the dynamic response of an inclined homogeneous Euler-Bernoulli beam due to a moving mass. The property matrices of the moving mass element are derived by taking into account of the effects of inertial force, Coriolis force and centrifugal force induced by a moving mass. Mamandi and Kargarnovin [17] studied dynamic behavior of inclined pinned-pinned Timoshenko beams made of linear, homogenous and isotropic material subjected to a traveling mass/force. The inertial force due to the motion of the traveling mass on the deformed shape of the beam is considered. Bahmyari et al. [18] presented the finite element dynamic analysis of inclined composite laminated beams under a moving distributed mass with constant speed. The algorithm developed accounts for inertial, Coriolis, and centrifugal forces due to the moving distributed mass and friction force between the beam and the moving distributed mass.

According to authors' best knowledge, there have not been any studies on dynamic analysis of inclined FGSW beams subjected to moving mass reported in the literature so far. In this paper, dynamic analysis of an inclined FGSW beam subjected to traveling mass is studied using a moving mass element. The beam is composed of a fully ceramic core and two skin layers of FGM. The material properties of the FGM skin layers are assumed to vary continuously through the thickness of the beam according to a powerlaw. Mori-Tanaka scheme is employed to evaluate the effective properties. The effects of interaction forces due to the action of the traveling mass including the inertia force, Coriolis force and centrifugal force are considered. The overall matrices are received by 
adding the contribution of the mass, damping and stiffness matrices of the moving mass element, respectively. The present work focuses on the use of hierarchical functions as interpolation functions to derive a finite element formulation for the analysis. Numerical investigation is carried out to show the effects of the material gradient index, layer thickness ratio, inclined angle as well as the weight of the moving mass and its velocity on dynamic responses of FGSW beam.

\section{THEORETICAL FORMULATION}

An inclined FGSW beam element with length $l$, width $b$ and height $h$, traveled by a moving mass $m_{c}$ as shown in Fig. 1 is considered. The beam element is inclined an angle $\beta$ to the horizontal plane. The local coordinate $(x, z)$ is chosen such that the $x$-axis is on the mid-plane, and the $z$-axis is perpendicular to the mid-plane and directs upward.

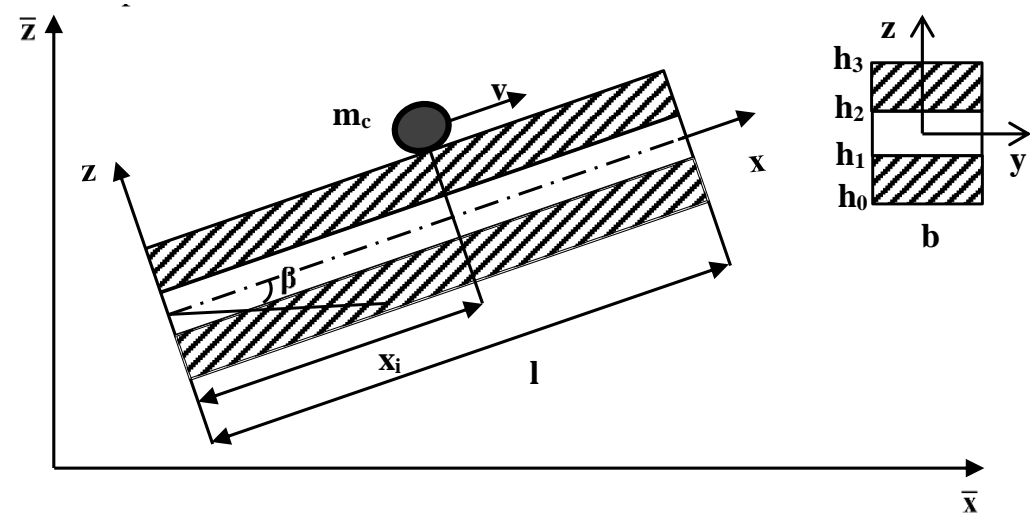

Fig. 1. An inclined FGSW beam element traveled by a moving mass $m_{c}$

The beam element is composed of a fully ceramic core and two skin layers of transverse FGM. The vertical positions of the bottom, top and of the two interfaces between the layers are denoted by $h_{0}=-\frac{h}{2}, h_{1}, h_{2}, h_{3}=\frac{h}{2}$. The volume fraction function $V_{c}^{(k)}$ of ceramic at the $k^{\text {th }}$ layer is given by [5]

$$
\begin{cases}V_{c}^{(1)}(z)=\left(\frac{z-h_{0}}{h_{1}-h_{0}}\right)^{n}, & z \in\left[h_{0}, h_{1}\right] \\ V_{c}^{(2)}(z)=1 & , z \in\left[h_{1}, h_{2}\right] \\ V_{c}^{(3)}(z)=\left(\frac{z-h_{3}}{h_{2}-h_{3}}\right)^{n}, & z \in\left[h_{2}, h_{3}\right]\end{cases}
$$

where $n$ is a non-negative material grading index.

This paper employs Mori-Tanaka scheme to evaluate the effective material properties. According to the Mori-Tanaka scheme, the effective local bulk modulus $K_{f}^{(k)}$ and the 
shear modulus $G_{f}^{(k)}$ of the $k^{\text {th }}$ layer of the sandwich beams can be given by [9]

$$
\begin{aligned}
& \frac{K_{f}^{(k)}-K_{m}^{(k)}}{K_{c}^{(k)}-K_{m}^{(k)}}=\frac{V_{c}^{(k)}}{1+\left(1-V_{c}^{(k)}\right)\left(K_{c}^{(k)}-K_{m}^{(k)}\right) /\left(K_{m}^{(k)}+4 G_{m}^{(k)} / 3\right)}, \\
& \frac{G_{f}^{(k)}-G_{m}^{(k)}}{G_{c}^{(k)}-G_{m}^{(k)}}=\frac{V_{c}^{(k)}}{1+\left(1-V_{c}^{(k)}\right)\left(G_{c}^{(k)}-G_{m}^{(k)}\right) /\left\{G_{m}^{(k)}+G_{m}^{(k)}\left(9 K_{m}^{(k)}+8 G_{m}^{(k)}\right) /\left[6\left(K_{m}^{(k)}+2 G_{m}^{(k)}\right)\right]\right\}},
\end{aligned}
$$

where

$$
K_{c}^{(k)}=\frac{E_{c}^{(k)}}{3\left(1-2 \mu_{c}^{(k)}\right)}, G_{c}^{(k)}=\frac{E_{c}^{(k)}}{2\left(1+\mu_{c}^{(k)}\right)}, K_{m}^{(k)}=\frac{E_{m}^{(k)}}{3\left(1-2 \mu_{m}^{(k)}\right)}, G_{m}^{(k)}=\frac{E_{m}^{(k)}}{2\left(1+\mu_{m}^{(k)}\right)},
$$

are the local bulk modulus and the shear modulus of the ceramic and metal at the $k^{\text {th }}$ layer, respectively.

Noting that the effective mass density $\rho_{f}^{(k)}$ is defined by Voigt model as [9]

$$
\rho_{f}^{(k)}=\left(\rho_{c}^{(k)}-\rho_{m}^{(k)}\right) V_{c}^{(k)}+\rho_{m}^{(k)} .
$$

The effective Young's modulus $E_{f}^{(k)}$ and Poisson's ratio $v_{f}^{(k)}$ are computed via effective bulk modulus and shear modulus as

$$
E_{f}^{(k)}=\frac{9 K_{f}^{(k)} G_{f}^{(k)}}{3 K_{f}^{(k)}+G_{f}^{(k)}}, \quad v_{f}^{(k)}=\frac{3 K_{f}^{(k)}-2 G_{f}^{(k)}}{6 K_{f}^{(k)}+2 G_{f}^{(k)}} .
$$

Based on the first-order shear deformation beam theory, the displacements in $x$ - and $z$-directions, $u_{1}(x, z, t)$ and $u_{3}(x, z, t)$, respectively, at any point of the inclined beam element are given by

$$
u_{1}(x, z, t)=u(x, t)-z \theta(x, t), \quad u_{3}(x, z, t)=w(x, t),
$$

where $z$ is the distance from the mid-plane to the considering point; $u(x, t)$ and $w(x, t)$ are, respectively, the displacements of the point on the mid-plane in $x$ - and $z$-directions; $\theta(x, t)$ is the cross-sectional rotation.

The axial strain $\left(\varepsilon_{x x}\right)$ and the shear strain $\left(\gamma_{x z}\right)$ resulted from Eq. (7) are of the forms

$$
\varepsilon_{x x}=u_{, x}-z \theta, x, \quad \gamma_{x z}=w_{, x}-\theta,
$$

where a subscript comma is used to indicate the derivative of the variable with respect to the spatial coordinate $x$, that is $(.)_{, x}=\partial(.) / \partial x$.

Based on the Hooke's law, the constitutive relation for the FGSW beam element is as follows

$$
\sigma_{x x}^{(k)}=E_{f}^{(k)}(z) \varepsilon_{x x}, \quad \tau_{x z}^{(k)}=\psi G_{f}^{(k)}(z) \gamma_{x z}
$$

where $\sigma_{x x}^{(k)}$ and $\tau_{x z}^{(k)}$ are the axial stress and shear stress at the $k^{\text {th }}$ layer, respectively; $\psi$ is the shear correction factor, equals to $5 / 6$ for the beams with rectangular cross-section considered herein. 
The strain energy of the beam element $\left(U_{e}\right)$ resulted from Eq. (8) and Eq. (9) is

$$
U_{e}=\frac{1}{2} \int_{0}^{l} \int_{A}\left(\sigma_{x x}^{(k)} \varepsilon_{x x}+\tau_{x z}^{(k)} \gamma_{x z}\right) \mathrm{d} A \mathrm{~d} x=\frac{1}{2} \int_{0}^{l}\left[A_{11} u_{, x}^{2}-2 A_{12} u_{, x} \theta_{, x}+A_{22} \theta_{, x}^{2}+\psi A_{33}\left(w_{, x}-\theta\right)^{2}\right] \mathrm{d} x .
$$

The kinetic energy resulted from Eq. (7) is of the form

$$
\mathcal{T}_{e}=\frac{1}{2} \int_{0}^{l} \int_{A} \rho_{f}^{(k)}(z)\left(\dot{u}_{1}^{2}+\dot{u}_{3}^{2}\right) \mathrm{d} A \mathrm{~d} x=\frac{1}{2} \int_{0}^{l}\left[I_{11} \dot{u}^{2}+I_{11} \dot{w}^{2}-2 I_{12} \dot{u} \dot{\theta}+I_{22} \dot{\theta}^{2}\right] \mathrm{d} x,
$$

where the overhead dot (.) indicates derivative with respect to time $t$. In Eqs. (10) and (11), $A$ is the cross-sectional area; $A_{11}, A_{12}, A_{22}$ and $A_{33}$ are, respectively, the extensional, extensional-bending coupling, bending rigidities and the shear rigidity, which are defined as

$$
\left(A_{11}, A_{12}, A_{22}\right)=b \sum_{k=1}^{3} \int_{h_{k-1}}^{h_{k}} E_{f}^{(k)}(z)\left(1, z, z^{2}\right) \mathrm{d} z, \quad A_{33}=b \sum_{k=1}^{3} \int_{h_{k-1}}^{h_{k}} G_{f}^{(k)}(z) \mathrm{d} z,
$$

and $I_{11}, I_{12}, I_{22}$ are the mass moments, defined as

$$
\left(I_{11}, I_{12}, I_{22}\right)=b \sum_{k=1}^{3} \int_{h_{k-1}}^{h_{k}} \rho_{f}^{(k)}(z)\left(1, z, z^{2}\right) \mathrm{d} z
$$

\section{FINITE ELEMENT FORMULATION}

The finite element formulation for dynamic analysis of the beam is derived in this section by using hierarchical functions to interpolate the kinematic variables. These shape functions are of the forms [19]

$$
N_{1}=\frac{1}{2}(1-\xi), \quad N_{2}=\frac{1}{2}(1+\xi), \quad N_{3}=\left(1-\xi^{2}\right), N_{4}=\xi\left(1-\xi^{2}\right),
$$

with $\xi=2 \frac{x}{l}-1$ being the natural coordinate.

The beam element based on the hierarchical functions needs middle values of the variables, and this increases the number of degrees of freedom of the element. In order to improve the efficiency of the element, the shear strain is constrained to be constant [20] for reducing the number of degrees of freedom. Using this procedure, the vector of nodal displacements for a generic element (d) has seven components as

$$
\mathbf{d}=\left\{\begin{array}{lllllll}
u_{1} & u_{2} & w_{1} & \theta_{1} & \theta_{3} & w_{2} & \theta_{2}
\end{array}\right\}^{T} .
$$


In the above equation and hereafter, the superscript ' $T$ ' is used to denote the transpose of a vector or a matrix. By constraining the shear strain to constant, the displacements and rotation are interpolated as [21]

$$
\begin{aligned}
& u=\frac{1}{2}(1-\xi) u_{1}+\frac{1}{2}(1+\xi) u_{2} \\
& \theta=\frac{1}{2}(1-\xi) \theta_{1}+\frac{1}{2}(1+\xi) \theta_{2}+\left(1-\xi^{2}\right) \theta_{3} \\
& w=\frac{1}{2}(1-\xi) w_{1}+\frac{1}{2}(1+\xi) w_{2}+\frac{l}{8}\left(1-\xi^{2}\right)\left(\theta_{1}-\theta_{2}\right)+\frac{l}{6} \xi\left(1-\xi^{2}\right) \theta_{3} .
\end{aligned}
$$

In matrix forms, we can write Eq. (16) in the forms

$$
u=\mathbf{N}_{u} \mathbf{d}, w=\mathbf{N}_{w} \mathbf{d}, \theta=\mathbf{N}_{\theta} \mathbf{d}
$$

where

$$
\begin{aligned}
& \mathbf{N}_{u}=\left\{\begin{array}{lllllll}
N_{1} & N_{2} & 0 & 0 & 0 & 0 & 0
\end{array}\right\}^{T}, \\
& \mathbf{N}_{\theta}=\left\{\begin{array}{lllllll}
0 & 0 & 0 & N_{1} & N_{3} & 0 & N_{2}
\end{array}\right\}^{T}, \\
& \mathbf{N}_{w}=\left\{\begin{array}{lllllll}
0 & 0 & N_{1} & \frac{l}{8} N_{3} & \frac{l}{6} N_{4} & N_{2} & -\frac{l}{8} N_{3}
\end{array}\right\}^{T},
\end{aligned}
$$

with $N_{1}, N_{2}, N_{3}, N_{4}$ are defined by Eq. (14). From the displacement field in Eq. (17), one can rewrite the strain energy (10) in the form

$$
U_{e}=\frac{1}{2} \mathbf{d}^{T} \mathbf{k} \mathbf{d}, \quad \text { with } \mathbf{k}=\mathbf{k}_{u u}+\mathbf{k}_{u \theta}+\mathbf{k}_{\theta \theta}+\mathbf{k}_{s},
$$

where $\mathbf{k}$ is the element stiffness matrix; $\mathbf{k}_{u u}, \mathbf{k}_{u \theta}, \mathbf{k}_{\theta \theta}$ and $\mathbf{k}_{s}$ are, respectively, the stiffness matrices stemming from the axial stretching, axial stretching-bending coupling, bending and shear deformation. Using $(.)_{, \xi}=\frac{l}{2}(.)_{, x} ;(.)_{, \xi \xi}=\frac{l^{2}}{4}(.)_{, x x} ; \mathrm{d} \xi=\frac{2}{l} \mathrm{~d} x$, these matrices have the following forms

$$
\begin{aligned}
& \mathbf{k}_{u u}=\int_{0}^{l} \mathbf{N}_{u, x}^{T} A_{11} \mathbf{N}_{u, x} \mathrm{~d} x, \quad \mathbf{k}_{u \theta}=-\int_{0}^{l} \mathbf{N}_{u, x}^{T} A_{12} \mathbf{N}_{\theta, x} \mathrm{~d} x, \\
& \mathbf{k}_{\theta \theta}=\int_{0}^{l} \mathbf{N}_{\theta, x}^{T} A_{22} \mathbf{N}_{\theta, x} \mathrm{~d} x, \quad \mathbf{k}_{s}=\psi \int_{0}^{l}\left(\mathbf{N}_{w, x}^{T}-\mathbf{N}_{\theta}^{T}\right) A_{33}\left(\mathbf{N}_{w, x}-\mathbf{N}_{\theta}\right) \mathrm{d} x .
\end{aligned}
$$

Similarly, the kinetic energy (11) can also be written in the form

$$
\mathcal{T}_{e}=\frac{1}{2} \dot{\mathbf{d}}^{T} \mathbf{m} \dot{\mathbf{d}} \quad \text { with } \mathbf{m}=\mathbf{m}_{u u}+\mathbf{m}_{u \theta}+\mathbf{m}_{\theta \theta}+\mathbf{m}_{w w},
$$


where $\mathbf{m}$ denotes the element mass matrix, and

$$
\begin{array}{ll}
\mathbf{m}_{u u}=\int_{0}^{l} \mathbf{N}_{u}^{T} I_{11} \mathbf{N}_{u} \mathrm{~d} x, \quad \mathbf{m}_{w w}=\int_{0}^{l} \mathbf{N}_{w}^{T} I_{11} \mathbf{N}_{w} \mathrm{~d} x, \\
\mathbf{m}_{u \theta}=-\int_{0}^{l} \mathbf{N}_{u}^{T} I_{12} \mathbf{N}_{\theta} \mathrm{d} x, & \mathbf{m}_{\theta \theta}=\int_{0}^{l} \mathbf{N}_{\theta}^{T} I_{22} \mathbf{N}_{\theta} \mathrm{d} x,
\end{array}
$$

are, respectively, the element mass matrices resulted from the axial and transverse translations, axial translation-rotation coupling, cross-sectional rotation.

When beam is inclined an angle $\beta$ to the horizontal plane as in Fig. 1, the displacement components of an arbitrary point on the inclined beam in the local $x$ and $z$ directions, $u$ and $w$ are related to those in the global $\bar{x}$ and $\bar{z}$ directions, $\bar{u}$ and $\bar{w}$

$$
\bar{u}=u \cos \beta-w \sin \beta ; \quad \bar{w}=u \sin \beta+w \cos \beta .
$$

Because the local rotations and the global ones are identical, the vector of local degrees of freedom $\mathbf{d}$ is related to the global one $\overline{\mathbf{d}}$ by $\mathbf{d}=\mathbf{T} \overline{\mathbf{d}}$ where $\overline{\mathbf{d}}=\left\{\begin{array}{lllllll}\bar{u}_{1} & \bar{u}_{2} & \bar{w}_{1} & \bar{\theta}_{1} & \bar{\theta}_{3} & \bar{w}_{2} & \bar{\theta}_{2}\end{array}\right\}^{T}$ and

$$
\mathbf{T}=\left[\begin{array}{ccccccc}
\cos \beta & 0 & \sin \beta & 0 & 0 & 0 & 0 \\
0 & \cos \beta & 0 & 0 & 0 & \sin \beta & 0 \\
-\sin \beta & 0 & \cos \beta & 0 & 0 & 0 & 0 \\
0 & 0 & 0 & 1 & 0 & 0 & 0 \\
0 & 0 & 0 & 0 & 1 & 0 & 0 \\
0 & -\sin \beta & 0 & 0 & 0 & \cos \beta & 0 \\
0 & 0 & 0 & 0 & 0 & 0 & 1
\end{array}\right]
$$

is the transformation matrix between the local coordinate and the global one.

The global element stiffness and mass matrices are finally computed as

$$
\overline{\mathbf{k}}=\mathbf{T}^{T} \mathbf{k T} \text { and } \overline{\mathbf{m}}=\mathbf{T}^{T} \mathbf{m} \mathbf{T},
$$

with $\mathbf{k}$ and $\mathbf{m}$ are given in Eqs. (19) and (21). The structural mass matrix $\overline{\mathbf{M}}_{b}$ and stiffness matrix $\overline{\mathbf{K}}_{b}$ of the inclined FGSW beam are obtained by assembling the corresponding element matrices over the total elements.

Assumption that the moving mass $m_{c}$ is located at point $i$ of the beam element. The interaction forces in the $x$ - and $z$-directions due to the action of the traveling mass are respectively given by [16]

$$
F_{x}=m_{c} \ddot{u}_{c}, F_{z}=m_{c}\left(\ddot{w}_{c}+2 v \dot{w}_{c, x}+v^{2} w_{c, x x}\right),
$$

where $v$ is the velocity of the moving mass; $u_{c}, w_{c}$ represent the displacement components of the contact point $i$ in the local $x$ and $z$ directions of the beam element, respectively; $m_{c} \ddot{u}_{c}, m_{c} \ddot{w}_{c}$ represent the inertia forces; and $2 m_{c} v \dot{w}_{c, x}, m_{c} v^{2} w_{c, x x}$ represent the Coriolis force and centrifugal force, respectively. The equivalent nodal forces of the beam element induced by the two forces given by Eq. (26) are [16]

$$
f_{k}=N_{u k} F_{x} \quad(k=1,2), \quad f_{k}=N_{w k} F_{z} \quad(k=3,4,5,6,7),
$$


where $N_{u k}, N_{w k}$ are the hierarchical functions defined in Eq. (18). The displacement components of the contact point $i$ can be also interpolated from the nodal displacements as

$$
u_{c}=N_{u 1} u_{1}+N_{u 2} u_{2}, \quad w_{c}=N_{w 3} w_{1}+N_{w 4} \theta_{1}+N_{w 5} \theta_{3}+N_{w 6} w_{2}+N_{w 7} \theta_{2} .
$$

From Eq. (28), one can receive the time derivatives of displacement components, then substituting into Eqs. (26), (27), and writing the resulting expressions in matrix form yield

$$
\mathbf{f}_{c}=\mathbf{m}_{c} \ddot{\mathbf{d}}+\mathbf{c}_{c} \dot{\mathbf{d}}+\mathbf{k}_{c} \mathbf{d},
$$

with $\mathbf{d}$ is given in Eq. (15). In Eq. (29),

$$
\begin{aligned}
& \mathbf{m}_{c}=m_{c}\left[\begin{array}{ccccccc}
N_{1}^{2} & N_{1} N_{2} & 0 & 0 & 0 & 0 & 0 \\
N_{1} N_{2} & N_{2}^{2} & 0 & 0 & 0 & 0 & 0 \\
0 & 0 & N_{1}^{2} & \frac{l}{8} N_{1} N_{3} & \frac{l}{6} N_{1} N_{4} & N_{1} N_{2} & -\frac{l}{8} N_{1} N_{3} \\
0 & 0 & \frac{l}{8} N_{1} N_{3} & \frac{l^{2}}{64} N_{3}^{2} & \frac{l^{2}}{48} N_{3} N_{4} & \frac{l}{8} N_{2} N_{3} & -\frac{l^{2}}{64} N_{3}^{2} \\
0 & 0 & \frac{l}{6} N_{1} N_{4} & \frac{l^{2}}{48} N_{3} N_{4} & \frac{l^{2}}{36} N_{4}^{2} & \frac{l}{6} N_{2} N_{4} & -\frac{l^{2}}{48} N_{3} N_{4} \\
0 & 0 & N_{1} N_{2} & \frac{l}{8} N_{2} N_{3} & \frac{l}{6} N_{2} N_{4} & N_{2}^{2} & -\frac{l}{8} N_{2} N_{3} \\
0 & 0 & -\frac{l}{8} N_{1} N_{3} & -\frac{l^{2}}{64} N_{3}^{2} & -\frac{l^{2}}{48} N_{3} N_{4} & -\frac{l}{8} N_{2} N_{3} & \frac{l^{2}}{64} N_{3}^{2}
\end{array}\right], \\
& \mathbf{c}_{c}=2 m_{\mathcal{c}} \boldsymbol{v}\left[\begin{array}{ccccccc}
0 & 0 & 0 & 0 & 0 & 0 & 0 \\
0 & 0 & 0 & 0 & 0 & 0 & 0 \\
0 & 0 & N_{1} N_{1, x} & \frac{l}{8} N_{1} N_{3, x} & \frac{l}{6} N_{1} N_{4, x} & N_{1} N_{2, x} & -\frac{l}{8} N_{1} N_{3, x} \\
0 & 0 & \frac{l}{8} N_{1, x} N_{3} & \frac{l^{2}}{64} N_{3} N_{3, x} & \frac{l^{2}}{48} N_{3} N_{4, x} & \frac{l}{8} N_{2, x} N_{3} & -\frac{l^{2}}{64} N_{3} N_{3, x} \\
0 & 0 & \frac{l}{6} N_{1, x} N_{4} & \frac{l^{2}}{48} N_{3, x} N_{4} & \frac{l^{2}}{36} N_{4} N_{4, x} & \frac{l}{6} N_{2, x} N_{4} & -\frac{l^{2}}{48} N_{3, x} N_{4} \\
0 & 0 & N_{1, x} N_{2} & \frac{l}{8} N_{2} N_{3, x} & \frac{l}{6} N_{2} N_{4, x} & N_{2} N_{2, x} & -\frac{l}{8} N_{2} N_{3, x} \\
0 & 0 & -\frac{l}{8} N_{1, x} N_{3} & -\frac{l^{2}}{64} N_{3} N_{3, x} & -\frac{l^{2}}{48} N_{3} N_{4, x} & -\frac{l}{8} N_{2, x} N_{3} & \frac{l^{2}}{64} N_{3} N_{3, x}
\end{array}\right], \\
& \mathbf{k}_{c}=m_{c} v^{2}\left[\begin{array}{ccccccc}
0 & 0 & 0 & 0 & 0 & 0 & 0 \\
0 & 0 & 0 & 0 & 0 & 0 & 0 \\
0 & 0 & N_{1} N_{1, x x} & \frac{l}{8} N_{1} N_{3, x x} & \frac{l}{6} N_{1} N_{4, x x} & N_{1} N_{2, x x} & -\frac{l}{8} N_{1} N_{3, x x} \\
0 & 0 & \frac{l}{8} N_{1, x x} N_{3} & \frac{l^{2}}{64} N_{3} N_{3, x x} & \frac{l^{2}}{48} N_{3} N_{4, x x} & \frac{l}{8} N_{2, x x} N_{3} & -\frac{l^{2}}{64} N_{3} N_{3, x x} \\
0 & 0 & \frac{l}{6} N_{1, x x} N_{4} & \frac{l^{2}}{48} N_{3, x x} N_{4} & \frac{l^{2}}{36} N_{4} N_{4, x x} & \frac{l}{6} N_{2, x x} N_{4} & -\frac{l^{2}}{48} N_{3, x x} N_{4} \\
0 & 0 & N_{1, x x} N_{2} & \frac{l}{8} N_{2} N_{3, x x} & \frac{l}{6} N_{2} N_{4, x x} & N_{2} N_{2, x x} & -\frac{l}{8} N_{2} N_{3, x x} \\
0 & 0 & -\frac{l}{8} N_{1, x x} N_{3} & -\frac{l^{2}}{64} N_{3} N_{3, x x} & -\frac{l^{2}}{48} N_{3} N_{4, x x} & -\frac{l}{8} N_{2, x x} N_{3} & \frac{l^{2}}{64} N_{3} N_{3, x x}
\end{array}\right],
\end{aligned}
$$

are the mass, damping and stiffness matrices of the moving mass element written in the local coordinate system. It can be seen from Eqs. (30b), (30c) that the damping and 
stiffness matrices of the moving mass element are generated from transverse displacement only.

Using Eq. (23) one can also get

$$
\mathbf{u}_{c}=\mathbf{T} \overline{\mathbf{u}}_{c}, \mathbf{w}_{c}=\mathbf{T} \overline{\mathbf{w}}_{c} .
$$

Similarly, the nodal forces and the time derivatives of displacement components in local coordinate system can be also transformed into those in global coordinate system. Since, one receives

$$
\overline{\mathbf{f}}_{c}=\overline{\mathbf{m}}_{c} \ddot{\overrightarrow{\mathbf{d}}}+\overline{\mathbf{c}}_{c} \dot{\overline{\mathbf{d}}}+\overline{\mathbf{k}}_{c} \overline{\mathbf{d}}
$$

where

$$
\overline{\mathbf{m}}_{c}=\mathbf{T}^{T} \mathbf{m}_{c} \mathbf{T} ; \quad \overline{\mathbf{c}}_{c}=\mathbf{T}^{T} \mathbf{c}_{c} \mathbf{T} ; \quad \overline{\mathbf{k}}_{c}=\mathbf{T}^{T} \mathbf{k}_{c} \mathbf{T},
$$

are the mass, damping and stiffness matrices of the moving mass element written in global coordinate system, respectively.

The finite element equation for the dynamic analysis of the beam can be written in the form

$$
\overline{\mathbf{M}} \ddot{\overline{\mathbf{D}}}+\overline{\mathbf{C}} \dot{\overline{\mathbf{D}}}+\overline{\mathbf{K}} \overline{\mathbf{D}}=\overline{\mathbf{F}}^{e x},
$$

where $\overline{\mathbf{M}}, \overline{\mathbf{K}}$ are the instantaneous overall mass and stiffness matrices, respectively. They composed of the constant overall mass and stiffness matrices of the entire inclined beam itself and the time-dependent element property matrices of the moving mass element [16]. The instantaneous overall damping matrix $\overline{\mathbf{C}}$ is received by adding the damping matrix of the moving mass element $\overline{\mathbf{c}}_{c}$ to the damping matrix of the inclined beam itself $\overline{\mathbf{C}}_{b}$. The overall damping matrix $\overline{\mathbf{C}}_{b}$ of the inclined beam is proportional to the instantaneous overall mass and stiffness matrices by using the theory of Rayleigh damping [16].

The equivalent force vector $\mathbf{F}^{e x}$ has the following form

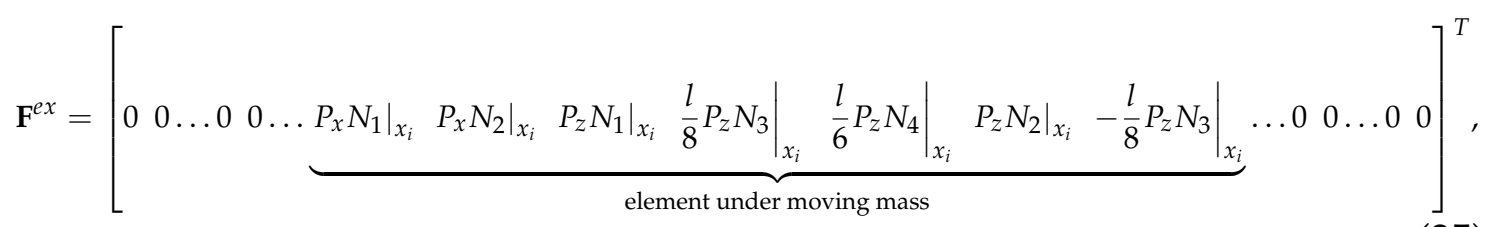

where $P_{x}, P_{z}$ are the corresponding force components of the equivalent force vector $\mathbf{P}$ induced by the $m_{c}$ at any time $t$. They are given by

$$
P_{x}=-m_{c} g \sin \beta, P_{z}=-m_{c} g \cos \beta,
$$

in which $g=9.81 \mathrm{~m} / \mathrm{s}^{2}$ is the acceleration of gravity. Noting that the effect of frictional force at the contact point $i$ between the moving mass and the inclined beam is small [16], and it is neglected in this paper. The local equivalent force vector in Eq. (35) must also transform into global coordinate to form the vector $\overline{\mathbf{F}}^{e x}$. The system of Eq. (34) can be solved by the direct integration Newmark method. The average acceleration method which ensures the unconditional convergence is adopted in the present work. 


\section{NUMERICAL RESULTS AND DISCUSSION}

The dynamic responses of a simply inclined supported FGSW beam subjected to a moving mass are numerically examined in this section. In the below, it is assumed that the core of the beam is pure $\mathrm{Si}_{3} \mathrm{~N}_{4}$ and FGM parts are composed of SUS304 and $\mathrm{Si}_{3} \mathrm{~N}_{4}$. The properties of these constituent materials are given in room temperature $(T=300 \mathrm{~K})$ as [22]:

- SUS304: $E_{m}=207.8 \mathrm{GPa} ; \rho_{m}=8166 \mathrm{~kg} / \mathrm{m}^{3} ; v_{m}=0.3$;

$-\mathrm{Si}_{3} \mathrm{~N}_{4}: E_{c}=322.3 \mathrm{GPa} ; \rho_{c}=2370 \mathrm{~kg} / \mathrm{m}^{3} ; v_{c}=0.3$.

Otherwise stated, an aspect ratio $L / h=20$ is assumed, where $L$ is the total length of the beam. To facilitate the discussion, the dynamic magnification factor $\left(D_{d}\right)$ is introduced as $D_{d}=\max \left(\frac{\bar{w}(L / 2, t)}{\bar{w}_{s t}}\right)$; where $\bar{w}_{s t}=m_{c} g L^{3} / 48 E_{m} I$ is the static deflection of a full metal beam under mid-span concentrated load of size $m_{c} g ; I$ is second moment of area of the cross-section. The weight of the moving mass is defined through mass ratio $m_{r}=m_{c} / \rho_{m} A L$, and the layer thickness ratio is defined using three number as (1-0-1), $(2-1-2),(1-1-1),(2-2-1),(1-2-1),(1-8-1)$, for example (1-1-1) means the thickness ratio of the bottom, core, and top layers is 1:1:1.

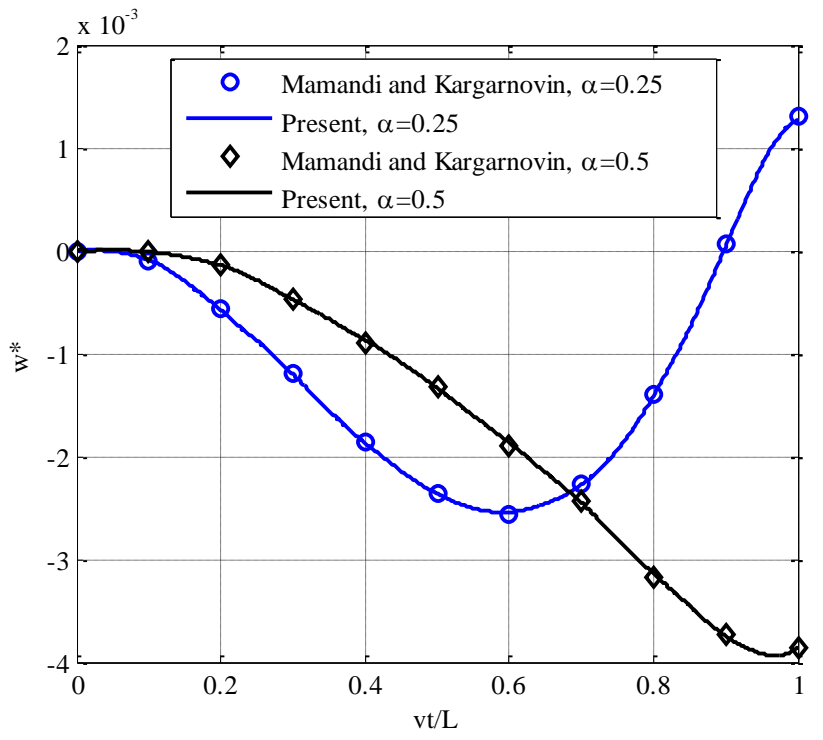

Fig. 2. Time histories for normalized mid-point deflection of homogenous beam

To confirm the convergence and accuracy of the derived formulation, we have to consider some special cases of this study to be compared with results in the literature. To this end, the time histories for normalized mid-point deflection of homogenous beam are compared with that of Mamandi and Kargarnovin [17] as shown in Fig. 2. In the figure, $w^{*}=\bar{w}(L / 2, t) / \bar{w}_{s t}$ is the dimensionless mid-span deflection; and the velocity ratio is defined according to in Ref. [17] as $\alpha=v / v_{c r}$, with $v_{c r}=(\pi / l) \sqrt{E I / \rho A}$ is the critical 
velocity of a moving force on a simply supported Euler-Bernoulli beam. It can be seen from the figure that the time histories received in this study are in good agreement with that of Ref. [17], regardless of the velocity ratio.

Tab. 1 compares the fundamental frequency parameters of a simply supported FGSW beam of the present paper with that of Ref. [9], where the modified Fourier series method is used. The fundamental frequency parameter is defined as $\mu=\frac{\omega L^{2}}{h} \sqrt{\rho_{m} / E_{m}}$, with $\omega$ is the fundamental natural frequency. Very good agreement between the results of the present work with that of Ref. [9] is noted from Tab. 1. It is worth mentioning that convergence of the results obtained in Fig. 2 and Tab. 1 has been achieved by using twenty elements, and this number of the elements will be used in the below computations.

Table 1. Comparison of fundamental frequency parameter of FGSW beam $(L / h=10)$

\begin{tabular}{cccccc}
\hline$n$ & Source & $(1-1-1)$ & $(1-2-1)$ & $(1-3-1)$ & $(1-4-1)$ \\
\hline \multirow{2}{*}{0} & Su et al. [9] & 5.3988 & 5.3988 & 5.3988 & 5.3988 \\
\cline { 2 - 6 } & Present & 5.3934 & 5.3934 & 5.3934 & 5.3934 \\
\hline \multirow{2}{*}{0.6} & Su et al. [9] & 3.7388 & 4.0246 & 4.2394 & 4.4004 \\
\cline { 2 - 6 } & Present & 3.7330 & 4.0187 & 4.2336 & 4.3946 \\
\hline \multirow{2}{*}{1} & Su et al. [9] & 3.4480 & 3.7782 & 4.0314 & 4.2220 \\
\cline { 2 - 6 } & Present & 3.4422 & 3.7723 & 4.0255 & 4.2162 \\
\hline \multirow{2}{*}{5} & Su et al. [9] & 2.9387 & 3.3101 & 3.6263 & 3.8709 \\
\cline { 2 - 6 } & Present & 2.9328 & 3.3040 & 3.6201 & 3.8649 \\
\hline
\end{tabular}

Tab. 2 lists the dynamic magnification factors of the beam with two values of the aspect ratio, $L / h=5$ and 20 , for various values of the grading index, the layer thickness ratio and the inclined angle of the beam. The velocity of the moving mass is taken by $v=20 \mathrm{~m} / \mathrm{s}$ and the mass ratio is $m_{r}=0.5$. Consider the case of $L / h=5$, it is clear that the factor $D_{d}$ increases as the grading index $n$ increases. The effect of the grading index on the factor $D_{d}$ can be explained by the dependence of the rigidities on this index. When the grading index increases, the beam contains more metal, and thus, its rigidities are lower, and this is the reason for the increases in the factor $D_{d}$ when raising $n$, no matter what the values of the layer thickness ratio and the inclined angle of the beam would be. On the contrary, the increase in the thickness of the core layer leads to the decrease in the factor $D_{d}$. This dependence is explained by the fact that for the present FGSW beam with ceramic hardcore, the rigidities of the beam are higher when the thickness of the core layer increases, and this leads to the factor $D_{d}$ decreases. In the case of $L / h=20$, the effect of the grading index, the layer thickness ratio and the inclined angle of the beam on the factor $D_{d}$ is similar to the case of $L / h=5$. That is, the factor $D_{d}$ of the FGSW beam increases as the grading index increases while it decreases as the layer thickness ratio and the inclined angle of the beam increase. The value of the factor $D_{d}$ is also dependent on 
Table 2. Variations of the dynamic magnification factor with the grading indexes, layer thickness ratio and inclined angle for $v=20 \mathrm{~m} / \mathrm{s}, m_{r}=0.5$

\begin{tabular}{|c|c|c|c|c|c|c|c|c|c|c|c|c|c|}
\hline \multirow{2}{*}{$\beta$} & \multirow{2}{*}{$n$} & \multicolumn{6}{|c|}{$L / h=5$} & \multicolumn{6}{|c|}{$L / h=20$} \\
\hline & & $(1-0-1)$ & $(2-1-2)$ & $(1-1-1)$ & $(2-2-1)$ & $(1-2-1)$ & $(1-8-1)$ & $(1-0-1)$ & $(2-1-2)$ & $(1-1-1)$ & $(2-2-1)$ & $(1-2-1)$ & $(1-8-1)$ \\
\hline \multirow{5}{*}{0} & 0 & 0.7299 & 0.7299 & 0.7299 & 0.7299 & 0.7299 & 0.7299 & 0.6557 & 0.6557 & 0.6557 & 0.6557 & 0.6557 & 0.6557 \\
\hline & 0.5 & 0.9137 & 0.9008 & 0.8707 & 0.8571 & 0.8509 & 0.7815 & 0.8326 & 0.8223 & 0.8125 & 0.8011 & 0.7908 & 0.7195 \\
\hline & 1 & 0.9986 & 0.9795 & 0.9371 & 0.9238 & 0.9096 & 0.8036 & 0.9531 & 0.9092 & 0.8681 & 0.8382 & 0.8327 & 0.7491 \\
\hline & 2 & 1.0802 & 1.0352 & 1.0099 & 0.9869 & 0.9527 & 0.8297 & 1.0306 & 0.9988 & 0.9591 & 0.9187 & 0.8839 & 0.7750 \\
\hline & 5 & 1.1172 & 1.1000 & 1.0599 & 1.0185 & 1.0094 & 0.8560 & 1.0598 & 1.0512 & 1.0236 & 0.9824 & 0.9528 & 0.7968 \\
\hline \multirow{5}{*}{$\frac{\pi}{12}$} & 0 & 0.7053 & 0.7053 & 0.7053 & 0.7053 & 0.7053 & 0.7053 & 0.6333 & 0.6333 & 0.6333 & 0.6333 & 0.6333 & 0.6333 \\
\hline & 0.5 & 0.8818 & 0.8702 & 0.8416 & 0.8267 & 0.8216 & 0.7551 & 0.8043 & 0.7942 & 0.7848 & 0.7736 & 0.7639 & 0.6950 \\
\hline & 1 & 0.9642 & 0.9 & 0.9 & 0.8 & 0.8 & 0. & 7 & 82 & 36 & 93 & 0.8043 & 0.7236 \\
\hline & 2 & 1.0438 & 1.0002 & 0.9754 & 0.9510 & 0.9210 & 0.8019 & 0.9955 & 0.9648 & 0.9264 & 0.8867 & 0.8538 & 0.7486 \\
\hline & 5 & 1.0784 & 1.0626 & 1.0238 & 0.9817 & 0.9744 & 0.8266 & 1.0236 & 1.0154 & 0.9887 & 0.9481 & 0.9204 & 0.7696 \\
\hline \multirow{5}{*}{$\frac{\pi}{4}$} & 0 & 0.5174 & 0.5174 & 0.5174 & 0.5174 & 0.5174 & 0.5174 & 0.4633 & 0.4633 & 0.4633 & 0.4633 & 0.4633 & 0.4633 \\
\hline & 0.5 & 0.6442 & 0.6359 & 0.6212 & 0.6002 & 0.5985 & 0.5532 & 0.5892 & 0.5811 & 0.5743 & 0.5656 & 0.5591 & 0.5090 \\
\hline & 1 & 0.7037 & 0.6909 & 0.6688 & 0.6445 & 0.6427 & 0.5667 & 0.6741 & 0.6432 & 0.6143 & 0.5913 & 0.5884 & 0.5298 \\
\hline & 2 & 0.7637 & 0.7341 & 0.7112 & 0.6905 & 0.6792 & 0.5891 & 0.7286 & 0.7064 & 0.6784 & 0.6480 & 0.6254 & 0.5480 \\
\hline & 5 & 0.7863 & 0.7772 & 0.7527 & 0.7132 & 0.7104 & 0.6012 & 0.7489 & 0.7431 & 0.7239 & 0.6926 & 0.6740 & 0.5633 \\
\hline
\end{tabular}

the change of the $L / h$. In particular, with the velocity value considered in Tab. $2, v=20$ $\mathrm{m} / \mathrm{s}$, the factor $D_{d}$ decreases as $L / h$ increases, however the reduction is negligible. In addition, it can be seen from Tab. 2 that for any values of the grading index and the layer thickness ratio, the factor $D_{d}$ decreases as the inclined angle of the beam increases. This phenomenon has been explained as follows. Since the axial stiffness of the beam is much higher than its transverse stiffness, the axial displacement is much smaller than the transverse one. In this case, the global displacement components in Eq. (23) can be approximated as $\bar{w} \approx w \cos \beta, \bar{u} \approx-w \sin \beta$. Thus, the value of $\bar{u}$ increases and the value of $\bar{w}$ decreases when the inclined angle of the beam increases. This leads to the decrease in the transverse response of the beam.

Tab. 3 shows the effect of grading indexes, the layer thickness ratio and the inclined angle of the beam on the dynamic magnification factor $D_{d}$ with a velocity $v=100 \mathrm{~m} / \mathrm{s}$. From Tab. 3, one can see that the rule of dependence of above dynamic parameters on the factor $D_{d}$ is similar to the case $v=20 \mathrm{~m} / \mathrm{s}$. However, the difference is that a higher value of the $L / h$, the factor $D_{d}$ increases more significantly. The dependence of the factor $D_{d}$ on the aspect ratio $L / h$ with two values of the velocity of the moving mass as seen in Tab. 2 and Tab. 3 shows the effect of the shear deformation on the dynamic behavior of the beam.

The effect of the layer thickness ratio and inclined angle of the beam on the normalized mid-span deflection is depicted in Fig. 3 for $n=1, v=30 \mathrm{~m} / \mathrm{s}, m_{r}=0.5$. In the 
Table 3. Variations of the dynamic magnification factor with the grading indexes, layer thickness ratio and inclined angle for $v=100 \mathrm{~m} / \mathrm{s}, m_{r}=0.5$

\begin{tabular}{|c|c|c|c|c|c|c|c|c|c|c|c|c|c|}
\hline \multirow{2}{*}{$\beta$} & \multirow{2}{*}{$n$} & \multicolumn{6}{|c|}{$L / h=5$} & \multicolumn{6}{|c|}{$L / h=20$} \\
\hline & & $(1-0-1)$ & $(2-1-2)$ & $(1-1-1)$ & $(2-2-1)$ & $(1-2-1)$ & $(1-8-1)$ & $(1-0-1)$ & $(2-1-2)$ & $(1-1-1)$ & $(2-2-1)$ & $(1-2-1)$ & $(1-8-1)$ \\
\hline \multirow{5}{*}{0} & 0 & 0.7144 & 0.7144 & 0.7144 & 0.7144 & 0.7144 & 0.7144 & 0.9282 & 0.9282 & 0.9282 & 0.9282 & 0.9282 & 0.9282 \\
\hline & 0.5 & 0.9379 & 0.9275 & 0.9134 & 0.8993 & 0.8850 & 0.7964 & 1.3811 & 1.3209 & 1.2716 & 1.2347 & 1.1988 & 1.0439 \\
\hline & 1 & 0.9966 & 0.9660 & 0.9604 & 0.9465 & 0.9374 & 0.8322 & 1.5802 & 1.5095 & 1.4428 & 1.3878 & 1.3380 & 1.0991 \\
\hline & 2 & 1.1254 & 1.0556 & 0.9926 & 0.9707 & 0.9696 & 0.8629 & 1.7323 & 1.6637 & 1.5957 & 1.5265 & 1.4705 & 1.1559 \\
\hline & 5 & 1.2109 & 1.1511 & 1.0900 & 1.0292 & 0.9827 & 0.8912 & 1.8392 & 1.7771 & 1.7111 & 1.6346 & 1.5866 & 1.2117 \\
\hline \multirow{5}{*}{$\frac{\pi}{12}$} & 0 & 0.6905 & 0.6905 & 0.6905 & 0.6905 & 0.6905 & 0.6905 & 0.8966 & 0.8966 & 0.8966 & 0.8966 & 0.8966 & 0.8966 \\
\hline & 0.5 & 0.9051 & 0.8952 & 0.8811 & 0.8669 & 0.8549 & 0.7699 & 1.3340 & 1.2759 & 1.2281 & 1.1919 & 1.1579 & 1.0081 \\
\hline & 1 & 0.9632 & 0.9324 & 0.9270 & 0.9122 & 0.9051 & 0.8039 & 1.5259 & 1.4579 & 1.3936 & 1.3394 & 1.2923 & 1.0617 \\
\hline & 2 & 1.0880 & 1.0200 & 0.9597 & 0.9358 & 0.9361 & 0.8341 & 1.6729 & 1.6066 & 1.5410 & 1.4729 & 1.4203 & 1.1164 \\
\hline & 5 & 1.1712 & 1.1124 & 1.0534 & 0.9909 & 0.9479 & 0.8610 & 1.7760 & 1.7161 & 1.6523 & 1.5767 & 1.5323 & 1.1703 \\
\hline \multirow{5}{*}{$\frac{\pi}{4}$} & 0 & 0.5134 & 0.5134 & 0.5134 & 0.5134 & 0.5134 & 0.5134 & 0.6562 & 0.6562 & 0.6562 & 0.6562 & 0.6562 & 0.6562 \\
\hline & 0.5 & 0.6562 & 0.6501 & 0.6422 & 0.6317 & 0.6241 & 0.5661 & 0.9758 & 0.9337 & 0.8984 & 0.8703 & 0.8467 & 0.7373 \\
\hline & 1 & 0.7126 & 0.6743 & 0.6701 & 0.6607 & 0.6565 & 0.5894 & 1.1129 & 1.0653 & 1.0194 & 0.9780 & 0.9457 & 0.7770 \\
\hline & 2 & 0.8024 & 0.7547 & 0.7107 & 0.6763 & 0.6762 & 0.6107 & 1.2209 & 1.1718 & 1.1249 & 1.0734 & 1.0390 & 0.8167 \\
\hline & 5 & 0.8595 & 0.8206 & 0.7784 & 0.7243 & 0.7023 & 0.6279 & 1.2943 & 1.2523 & 1.2056 & 1.1467 & 1.1192 & 0.8558 \\
\hline
\end{tabular}

figures, $t^{*}=t / \Delta T$ with $\Delta T$ is the total time necessary for the mass crossing the beam. From the figure one can point out the dynamic deflection of the beam decreases as the layer thickness ratio increases, and this is explained by the increase in stiffness of the beam as mentioned above. Also, it can be observed again from Fig. 3 that the increase in the inclined angle of the beam leads to the decrease in the dynamic deflection. Thus, by increasing the inclined angle of the beam and the layer thickness ratio, it can be reduced the dynamic deflection.

In Fig. 4, the time histories for normalized mid-span deflection of the (1-2-1) beam are depicted for various values of the moving mass speed and mass ratio. The other parameters are given as: $\beta=\frac{\pi}{5}, n=1$. From Fig. 4 , it is clear that the velocity of the moving mass has a significant effect on both the dynamic deflection and the way the beam vibrates. For a given mass ratio, the beam performs more vibration cycles when the velocity is smaller. The values of the normalized mid-span deflection are also strongly influenced by the mass ratio. The dynamic deflection of the beam increases and reaches maximum value at a later time when the mass ratio increases.

In Fig. 5, the relation between the dynamic magnification factor $D_{d}$ and the moving mass velocity is illustrated with different mass ratio and inclined angle of the beam. As seen from the figure, the relation between $D_{d}$ and $v$ is similar to that of isotropic beams under a moving load, that is, the factor $D_{d}$ both increases and decreases when the velocity 

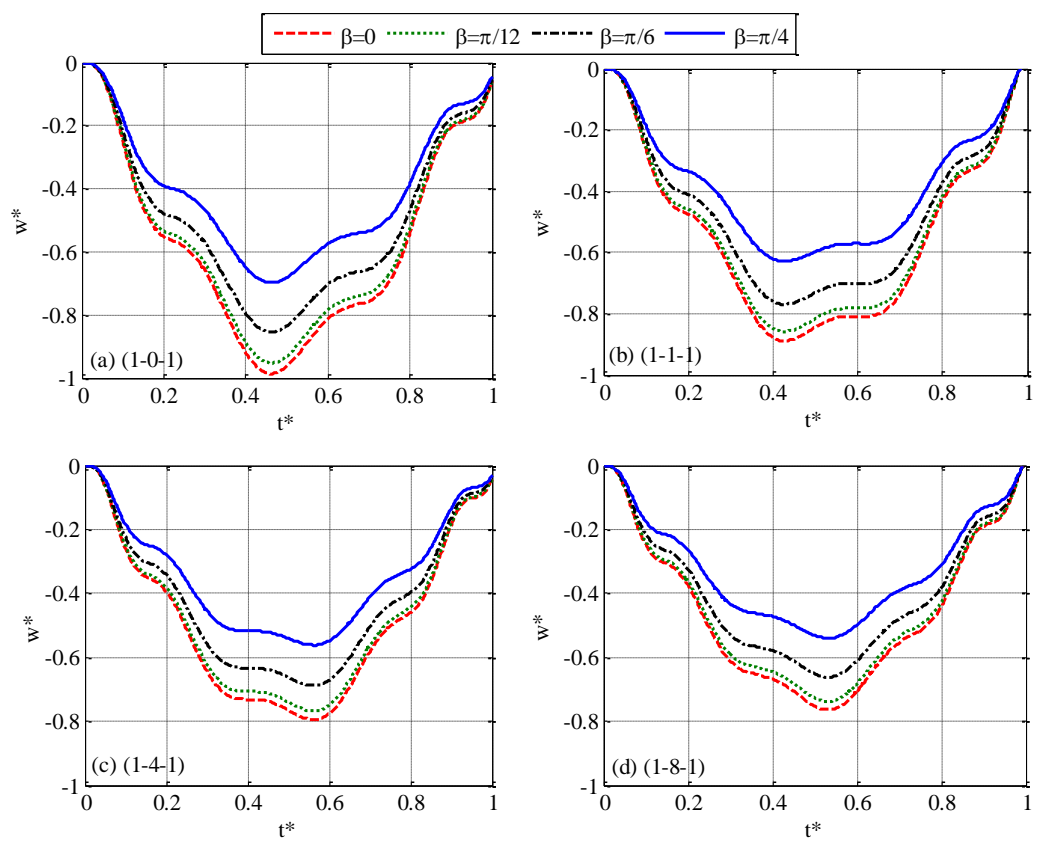

Fig. 3. Time histories for normalized mid-span deflection of beam with different layer thickness ratio and inclined angle of the beam: $n=1, v=30 \mathrm{~m} / \mathrm{s}, m_{r}=0.5$
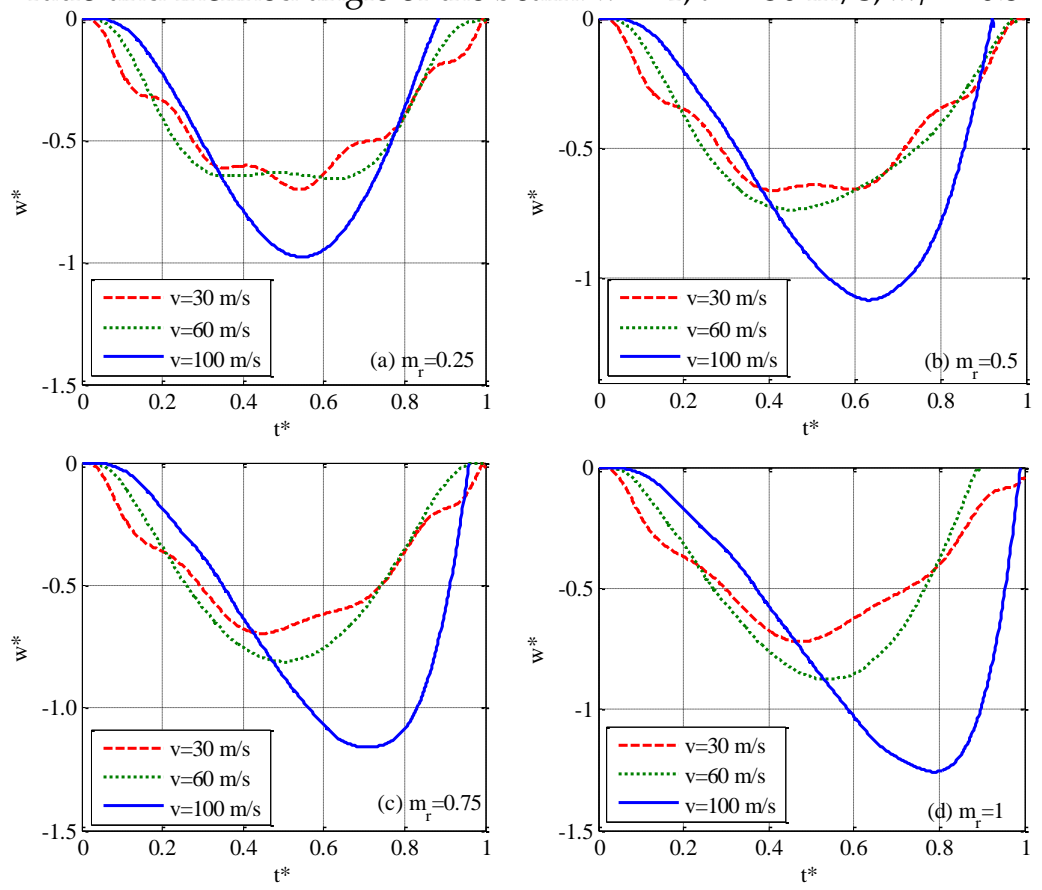

Fig. 4. Time histories for normalized mid-span deflection of (1-2-1) beam with different mass ratio and moving mass speed: $\beta=\frac{\pi}{5}, n=1$ 
of moving mass is low. When moving mass velocity increases, the factor $D_{d}$ increases and it reaches a maximum value. This dependency rule is true for any values of the mass ratio and inclined angle of the beam. In addition, the increase in the mass ratio leads to the decrease in the factor $D_{d}$ and the factor $D_{d}$ reaches the maximum value at the lower velocity of moving mass. Also, it is seen from this figure that the factor $D_{d}$ decreases as the inclined angle of the beam increases.
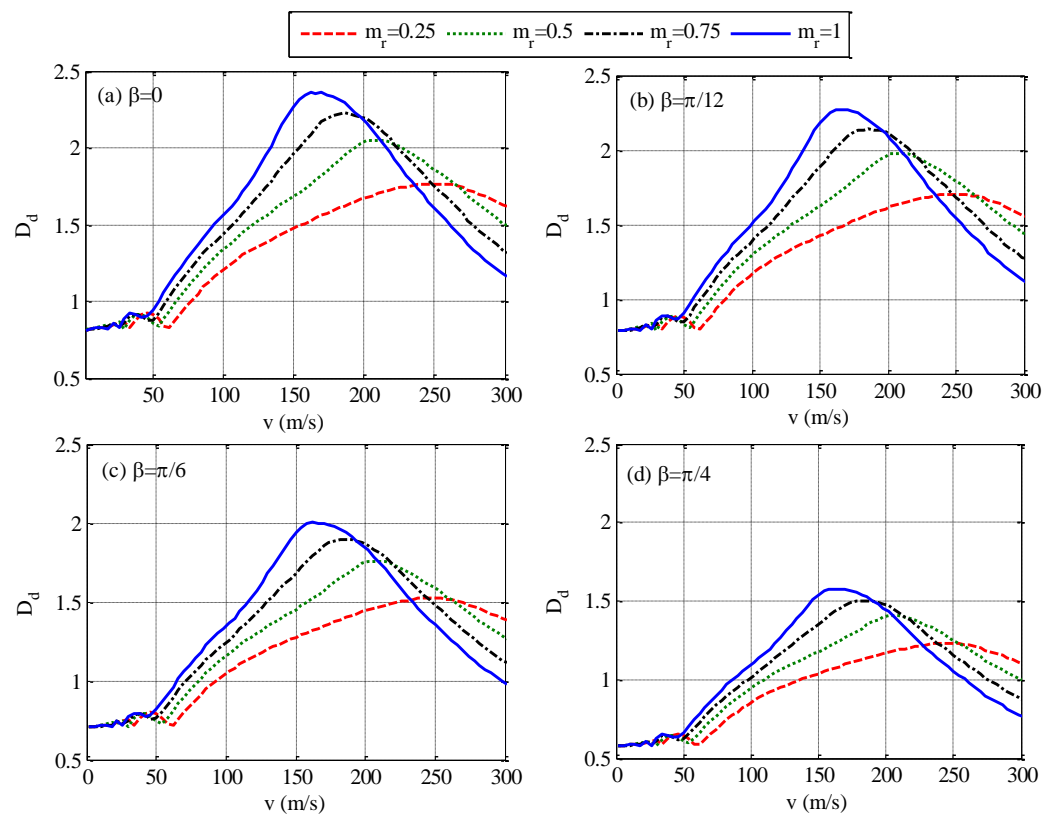

Fig. 5. Variation of the dynamic magnification factor of (1-2-1) beam with different mass ratio and inclined angle: $n=1$
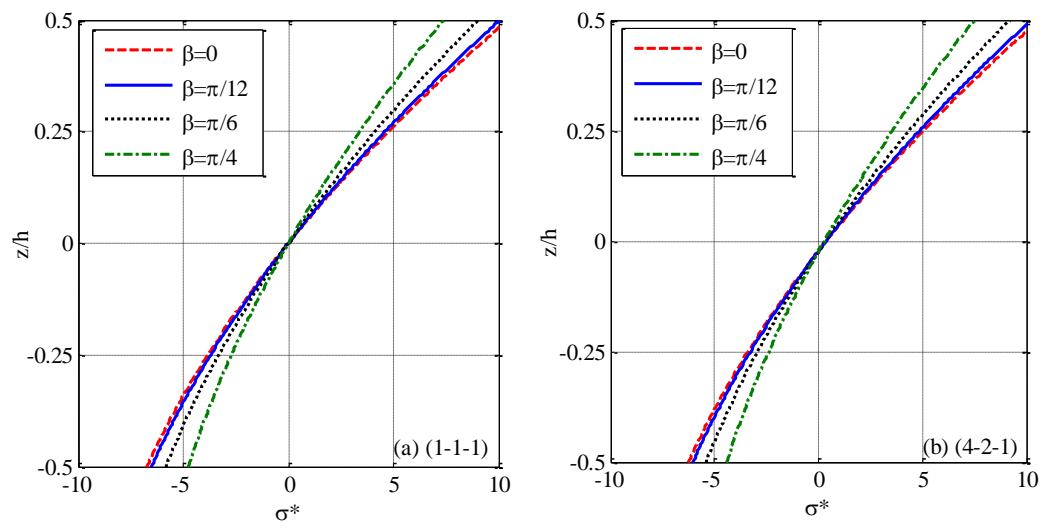

Fig. 6. Thickness distribution of normalized axial stress at mid-span section of inclined FGSW beam with different inclined angle: $v=30 \mathrm{~m} / \mathrm{s}, n=1, m_{r}=0.5$ 

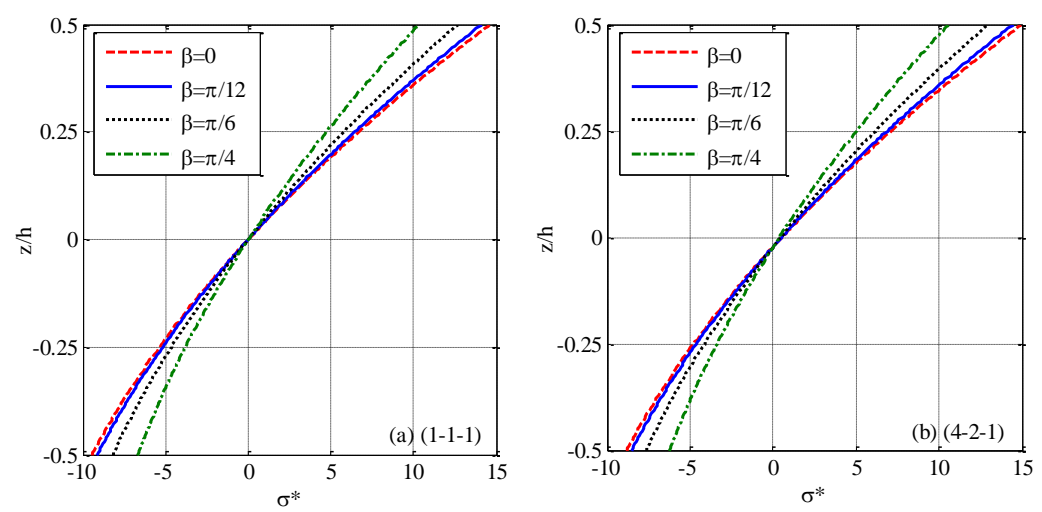

Fig. 7. Thickness distribution of normalized axial stress at mid-span section of inclined FGSW beam with different inclined angle: $v=100 \mathrm{~m} / \mathrm{s}, n=1, m_{r}=0.5$

In Fig. 6 and Fig. 7, the thickness distributions of the normalized axial stress at mid-span section of (1-1-1) beam and (4-2-1) beam are depicted for various values of inclined angle of the beam with $v=30 \mathrm{~m} / \mathrm{s}$ and $v=100 \mathrm{~m} / \mathrm{s}$, respectively. The stress in these figures was computed at the time when the moving mass arrives at the mid-span of the inclined beam, and it was normalized as $\sigma^{*}=\sigma_{x x} / \sigma_{0}$, where $\sigma_{0}=P L h / 8 I, P=$ $100 \mathrm{kN}$. At a given value of moving mass velocity, the maximum amplitude of both the compressive and tensile stresses decrease as the inclined angle of the beam increases. Thus, by raising the inclined angle of the beam, we could decrease not only the dynamic magnification factor, but also the maximum amplitude of the axial stress. Specially, it can be observed from these figures that in the case beam is unsymmetrical (Fig. 6(b), 7(b)), the stress does not vanish at the mid-span.

\section{CONCLUSION}

The dynamic analysis of an inclined FGSW beam subjected to moving mass is studied using the first-order shear deformation theory. The effective material properties of FGSW beam are estimated by Mori-Tanaka's scheme. The hierarchical functions are used to interpolate the displacements at the contact point $i$ between the moving mass and beam element, and these shape functions are also used to interpolate the kinematic variables of the beam. The theory of moving mass element has been used to establish the mass, damping and stiffness matrices of the moving mass element generated by the interaction forces including the inertia force, Coriolis force and centrifugal force. These matrices must be added to the corresponding ones of the entire inclined beam itself to receive the instantaneous overall mass, damping and stiffness matrices. The system of motion equations is solved with the aid of Newmark method. The accuracy of the derived formulation was validated by comparing the numerical results obtained in the present paper with the available data in the literature. The numerical results show a clear effect of the gradient index, the layer thickness ratio, moving mass speed, mass ratio and the inclined angle of the beam on the dynamic response of the beam. 


\section{ACKNOWLEDGMENTS}

This research is funded by Vietnam National Foundation for Science and Technology Development (NAFOSTED) under Grant Number 107.02-2018.23. The authors gratefully thank the Reviewers for their valuable comments and suggestions to improve the quality of the paper.

\section{REFERENCES}

[1] R. K. Bhangale and N. Ganesan. Thermoelastic buckling and vibration behavior of a functionally graded sandwich beam with constrained viscoelastic core. Journal of Sound and Vibration, 295, (1-2), (2006), pp. 294-316. https://doi.org/10.1016/j.jsv.2006.01.026.

[2] M. C. Amirani, S. M. R. Khalili, and N. Nemati. Free vibration analysis of sandwich beam with FG core using the element free Galerkin method. Composite Structures, 90, (3), (2009), pp. 373-379. https://doi.org/10.1016/j.compstruct.2009.03.023.

[3] T. Q. Bui, A. Khosravifard, C. Zhang, M. R. Hematiyan, and M. V. Golub. Dynamic analysis of sandwich beams with functionally graded core using a truly meshfree radial point interpolation method. Engineering Structures, 47, (2013), pp. 90-104. https://doi.org/10.1016/j.engstruct.2012.03.041.

[4] Y. Yang, C. C. Lam, K. P. Kou, and V. P. Iu. Free vibration analysis of the functionally graded sandwich beams by a meshfree boundary-domain integral equation method. Composite Structures, 117, (2014), pp. 32-39. https://doi.org/10.1016/j.compstruct.2014.06.016.

[5] T. P. Vo, H.-T. Thai, T.-K. Nguyen, A. Maheri, and J. Lee. Finite element model for vibration and buckling of functionally graded sandwich beams based on a refined shear deformation theory. Engineering Structures, 64, (2014), pp. 12-22. https://doi.org/10.1016/j.engstruct.2014.01.029.

[6] T. P. Vo, H.-T. Thai, T.-K. Nguyen, F. Inam, and J. Lee. A quasi-3D theory for vibration and buckling of functionally graded sandwich beams. Composite Structures, 119, (2015), pp. 1-12. https://doi.org/10.1016/j.compstruct.2014.08.006.

[7] T.-K. Nguyen, T. P. Vo, B.-D. Nguyen, and J. Lee. An analytical solution for buckling and vibration analysis of functionally graded sandwich beams using a quasi-3D shear deformation theory. Composite Structures, 156, (2016), pp. 238-252. https://doi.org/10.1016/j.compstruct.2015.11.074.

[8] T. P. Vo, H.-T. Thai, T.-K. Nguyen, F. Inam, and J. Lee. Static behaviour of functionally graded sandwich beams using a quasi-3D theory. Composites Part B: Engineering, 68, (2015), pp. 5974. https://doi.org/10.1016/j.compositesb.2014.08.030.

[9] Z. Su, G. Jin, Y. Wang, and X. Ye. A general Fourier formulation for vibration analysis of functionally graded sandwich beams with arbitrary boundary condition and resting on elastic foundations. Acta Mechanica, 227, (5), (2016), pp. 1493-1514. https://doi.org/10.1007/s00707016-1575-8.

[10] M. Şimşek and M. Al-shujairi. Static, free and forced vibration of functionally graded (FG) sandwich beams excited by two successive moving harmonic loads. Composites Part B: Engineering, 108, (2017), pp. 18-34. https://doi.org/10.1016/j.compositesb.2016.09.098.

[11] T. O. Awodola, S. A. Jimoh, and B. B. Awe. Vibration under variable magnitude moving distributed masses of non-uniform Bernoulli-Euler beam resting on Pasternak elastic foundation. Vietnam Journal of Mechanics, 41, (1), (2019), pp. 63-78. https://doi.org/10.15625/0866$7136 / 12781$. 
[12] A. O. Cifuentes. Dynamic response of a beam excited by a moving mass. Finite Elements in Analysis and Design, 5, (3), (1989), pp. 237-246. https://doi.org/10.1016/0168-874x(89)900462.

[13] E. Esmailzadeh and M. Ghorashi. Vibration analysis of a Timoshenko beam subjected to a travelling mass. Journal of Sound and Vibration, 199, (4), (1997), pp. 615-628. https://doi.org/10.1016/s0022-460x(96)99992-7.

[14] M. Şimşek. Vibration analysis of a functionally graded beam under a moving mass by using different beam theories. Composite Structures, 92, (4), (2010), pp. 904-917. https://doi.org/10.1016/j.compstruct.2009.09.030.

[15] I. Esen, M. A. Koc, and Y. Cay. Finite element formulation and analysis of a functionally graded Timoshenko beam subjected to an accelerating mass including inertial effects of the mass. Latin American Journal of Solids and Structures, 15, (10), (2018). https://doi.org/10.1590/1679-78255102.

[16] J.-J. Wu. Dynamic analysis of an inclined beam due to moving loads. Journal of Sound and Vibration, 288, (1-2), (2005), pp. 107-131. https://doi.org/10.1016/j.jsv.2004.12.020.

[17] A. Mamandi and M. H. Kargarnovin. Dynamic analysis of an inclined Timoshenko beam traveled by successive moving masses/forces with inclusion of geometric nonlinearities. Acta Mechanica, 218, (1-2), (2010), pp. 9-29. https://doi.org/10.1007/s00707-010-0400-z.

[18] E. Bahmyari, S. R. Mohebpour, and P. Malekzadeh. Vibration analysis of inclined laminated composite beams under moving distributed masses. Shock and Vibration, 2014, (2014), pp. 112. https://doi.org/10.1155/2014/750916.

[19] J. E. Akin. Finite elements for analysis and design. Academic Press, London, (1994).

[20] A. Tessler and S. B. Dong. On a hierarchy of conforming Timoshenko beam elements. Computers \& Structures, 14, (3-4), (1981), pp. 335-344. https://doi.org/10.1016/00457949(81)90017-1.

[21] D. K. Nguyen and T. T. Tran. Free vibration of tapered BFGM beams using an efficient shear deformable finite element model. Steel and Composite Structures, 29, (3), (2018), pp. 363-377. https://doi.org/10.12989/scs.2018.29.3.363.

[22] A. Fallah and M. M. Aghdam. Nonlinear free vibration and post-buckling analysis of functionally graded beams on nonlinear elastic foundation. European Journal of Mechanics - A/Solids, 30, (4), (2011), pp. 571-583. https://doi.org/10.1016/j.euromechsol.2011.01.005. 